'Sección de Reumatología, Hospital del Salvador, Universidad de Chile, Santiago, Chile.

${ }^{2}$ Departamento de Anatomía Patológica, Instituto Nacional del Tórax, Santiago, Chile.

Fuente de apoyo financiero: No hubo.

Recibido el 19 de enero de 2014 aceptado el 9 de octubre de 2014.

Correspondencia a: Daniel Erlij Avenida Salvador 364, Santiago, Chile. danerlij@gmail.com

\section{Fibrosarcoma de arteria pulmonar simulando un síndrome de Hughes Stovin}

\author{
DANIEL ERLIJ ${ }^{1}$, SUSANA MICHALLAND ${ }^{1}$, ÓSCAR NEIRA ${ }^{1}$, \\ CRISTINA FERNÁNDEZ ${ }^{2}$, VERÓNICA WOLFF ${ }^{1}$
}

\section{Pulmonary artery sarcoma mimicking a Hughes Stovin syndrome. Report of one case}

Pulmonary artery sarcoma is an uncommon entity with high mortality. Its clinical presentation is usually indistinguishable from pulmonary embolism, which leads to a significant delay in diagnosis. Hughes-Stovin syndrome is characterized by venous thrombosis and aneurysms of the pulmonary or bronchial artery. We report a 59 year-old female with a history of recurrent pulmonary embolism. In the last thromboembolic episode a pulmonary artery aneurysm was found on a CT scan. The patient was operated performing a left inferior lobectomy. The patient died five days after surgery and the pathological examination of the surgical piece revealed a pulmonary artery sarcoma.

(Rev Med Chile 2014; 142: 1478-1481)

Key words: Pulmonary embolism; Sarcoma; Vascular neoplasms.
$\mathrm{E}$ 1 fibrosarcoma de la arteria pulmonar (FAP) constituye una patología de baja frecuencia $y$ alta mortalidad, que muchas veces puede simular un tromboembolismo pulmonar (TEP $)^{1}$, lo cual la hace una entidad de difícil diagnóstico, el que se realiza en la mayoría de los casos de manera tardía ${ }^{2}$. El síndrome de Hughes Stovin (SHS) se caracteriza por trombosis venosas y aneurismas de la arteria pulmonar o bronquial ${ }^{3}$. Se presenta el caso de una paciente con antecedentes de TEP a repetición, derivada a cirugía para resolución de un aneurisma de arteria pulmonar. Si bien la sospecha clínica inicial fue de un SHS, la biopsia de la pieza operatoria evidenció un FAP como diagnóstico final.

\section{Caso clínico}

Mujer de raza blanca de 59 años, con antecedente de hipotiroidismo, comienzó su historia clínica en el año 2009, con un episodio de disnea y dolor torácico de inicio súbito con angio-CT compatible con un TEP, sin evidencia de trom- bosis venosa profunda (TVP). La paciente quedó con tratamiento anticoagulante oral con acenocumarol e INR entre 2 y 3 . El año 2010, presentó segundo episodio de TEP manifestado por disnea súbita, con INR en rango terapéutico y estudio de trombofilias negativo. El estudio de neoplasia oculta fue también negativo e incluyó CT de abdomen y pelvis, endoscopia digestiva alta, colonoscopia y evaluación ginecológica. El año 2011, sufrió un tercer episodio, con ecografía Doppler negativa nuevamente para TVP y ecocardiograma que evidenció presión sistólica de arteria pulmonar elevada en rango moderado $(53 \mathrm{mmHg})$. Se ajustó rango de INR para alcanzar valores entre 3 y 4. El 2012, se hospitalizó por cuarto episodio de disnea sugerente de nuevo TEP. En esta ocasión, refirió episodio de hemoptisis de escasa cuantía. El angio-CT (Figura 1) evidenció un aneurisma de la arteria interlobar izquierda de $4,1 \mathrm{~cm}$ de diámetro, asociado a aumento del diámetro del tronco de la arteria pulmonar. Fue evaluada por cirujanos de tórax para definir resolución de aneurisma y por reumatólogos, para evaluar patología de base que 
explique los hallazgos. Sus exámenes mostraron un hemograma normal y una velocidad de sedimentación eritrocitaria elevada en $44 \mathrm{~mm} / \mathrm{h}$, sin otras alteraciones. De la evaluación reumatológica destacó el antecedente de un aborto de primer trimestre con otros dos embarazos normales y una hija con lupus eritematoso sistémico en tratamiento. Los anticuerpos antinucleares fueron positivos a títulos de $1 / 80$, pero los anticuerpos anti-DNA, anticardiolipinas y el VDRL fueron negativos. El fondo de ojo no mostró hallazgos relevantes. Se propuso realizar un PET CT, que no alcanzó a realizarse. La evaluación por cirujanos de tórax definió resolución quirúrgica del aneurisma debido al antecedente de hemoptisis y el riesgo asociado de hemorragia severa con alta mortalidad. El ecocardiograma preoperatorio evidenció presión sistólica de arteria pulmonar de $55 \mathrm{mmHg}$, con insuficiencia tricuspídea severa secundaria y gran dilatación de cavidades derechas, además de severa dilatación de la rama izquierda de la arteria pulmonar. La coronariografía fue normal y el sondeo de cavidades derechas reveló dilatación de arterias pulmonares principales, oclusión de ramas lobares media e inferior derechas y dilatación aneurismática de rama lobar inferior izquierda con oclusión distal, con igual presión de arteria pulmonar que la indicada en el ecocardiograma. La paciente fue intervenida quirúrgicamente a pesar de las alteraciones descritas, debido a la alta mortalidad de un aneurisma complicado. Al no lograr la disección de las paredes del aneurisma del parénquima pulmonar, se realizó una lobectomía inferior izquierda. Se encontró dentro del aneurisma, gran cantidad de trombos. Al cierre de la toracotomía, presentó episodio de fibrilación ventricular, llevándose a cabo maniobras de reanimación y toracotomía exploradora que no evidenció complicaciones secundarias a la cirugía. Fue trasladada a la unidad de cuidados intensivos donde evolucionó en malas condiciones, con altos requerimientos de drogas vasoactivas, posteriormente sepsis y falla orgánica múltiple. Cinco días luego de la cirugía, presentó nuevo episodio de fibrilación ventricular que no respondió a maniobras de reanimación, por lo que fallece. El análisis de la pieza operatoria mostró una neoplasia en áreas fusadas, con acentuada celularidad y atipia, ubicada exclusivamente en las arterias de los ejes broncovasculares, ocupando los lúmenes de forma multifocal, sin distorsionar

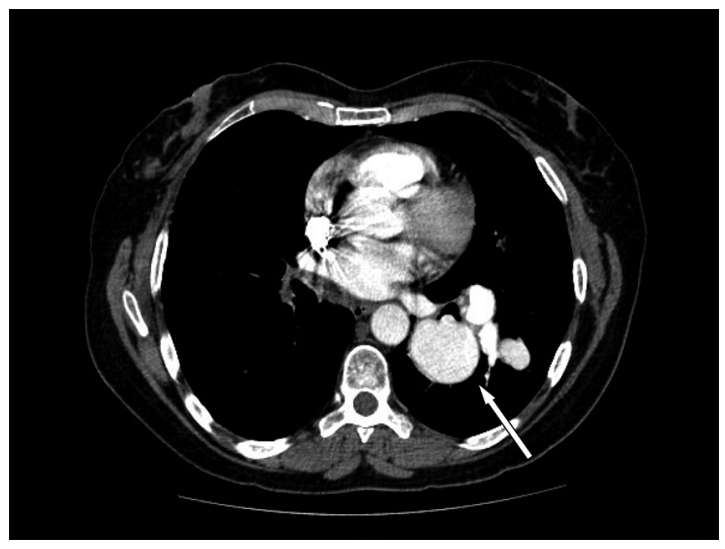

Figura 1. Angio- $C T$ de tórax mostrando aneurisma de la arteria interlobar izquierda (Flecha), más defectos de llene arteriales.

la arquitectura pulmonar y conservando la capa muscular de las mismas (Figura 2). El estudio inmunohistoquímico fue positivo únicamente para vimentina, siendo negativo para CD34, CD68, S100, actina, desmina y keratina AE1/AE3 y ALK1. Se concluye fibrosarcoma grado II de la íntima de la arteria pulmonar y de ramas secundarias. El supuesto trombo intraaneurismal correspondió a fragmentos de sarcoma.

\section{Discusión}

La presencia de aneurismas en la arteria pulmonar y sus ramas, en ausencia de comunicación arteriovenosa y de anomalías cardiacas, tiene como diagnóstico diferencial un número reducido de posibilidades, destacando las causas infecciosas como la sífilis y la tuberculosis, la hipertensión pulmonar severa, traumas y cuadros vasculíticos como la enfermedad de Behçet y el síndrome de Hughes Stovin ${ }^{3,4}$. Sumada la presencia de un TEP, las opciones se reducen a las últimas dos. La ausencia de úlceras orales y genitales en nuestra paciente hizo improbable la enfermedad de Behçet, por lo que la principal hipótesis diagnóstica fue el SHS. Esta es una entidad infrecuente, con aproximadamente 40 casos publicados desde su descripción en $1959^{5}$, caracterizada por la presencia de trombosis venosas periféricas y aneurismas de arterias pulmonares o bronquiales ${ }^{3}$. Actualmente no hay consenso en clasificarlo como una variante de la 

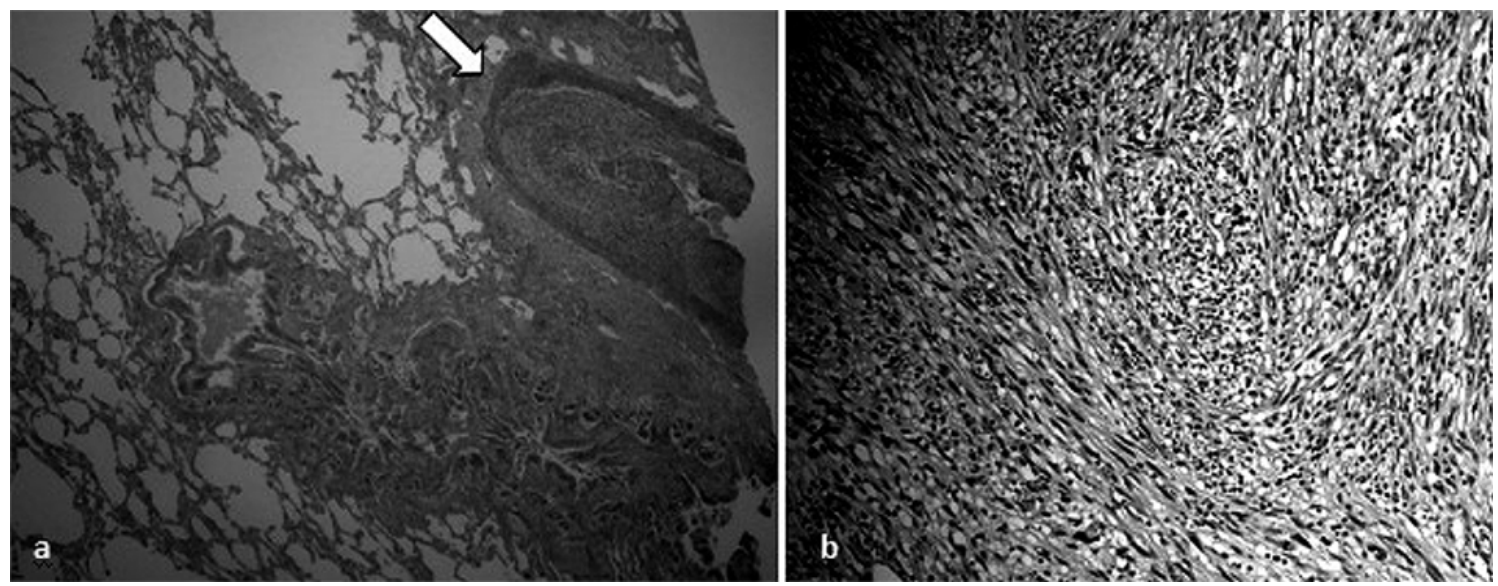

Figura 2. a. Eje broncovascular. Se observa bronquio y parenquima pulmonar normales y rama de la arteria pulmonar con neoplasia en el lumen (Flecha) (tinción HE, 40x). b. Neoplasia fusocelular en haces desordenados con acentuado pleomorfismo (tinción HE, 400x).

enfermedad de Behçet o como una entidad propia. Las trombosis venosas, relacionadas a fenómeno de tromboflebitis, suelen afectar a venas mayores y en $25 \%$ de los casos desarrollan tromboembolismo. Los aneurismas son generalmente múltiples, uni o bilaterales. El laboratorio es inespecífico ${ }^{3}$. Del punto de vista de imágenes el examen fundamental es el Angio-CT, destacando el aporte del PET-CT, que puede mostrar actividad inflamatoria en los aneurismas ${ }^{6}$. El tratamiento del SHS se basa en corticoides a altas dosis y ciclofosfamida, terapia que en algunos casos puede llevar a la resolución completa de los aneurismas. El uso de anticoagulación no se recomienda por el riesgo de sangrado con hemoptisis masiva, principal causa de muerte ${ }^{3,7}$. El rol de la cirugía es controversial, dado por la alta morbimortalidad y la recurrencia los sitios de la anastomosis, la cual puede llegar a $25 \%{ }^{3,8}$.

El fibrosarcoma de la arteria pulmonar se describió por primera vez en 1923 y, si bien es una entidad que tiene menos de 250 casos publicados en el mundo, su frecuencia es el doble de la del sarcoma intimal de la aorta ${ }^{9,-11}$. Suele presentarse generalmente entre los 45 y 55 años y con una discreta predilección por el género femenino ${ }^{12}$. Los síntomas pueden anteceder al diagnóstico en 3 a 12 meses, debido a la dificultad del mismo ${ }^{11}$, basada principalmente en su similitud a las manifestaciones clínicas y radiológicas del TEP. Esta situación explica más de la mitad de los errores diagnósticos relacionados a esta última ${ }^{13}$ y permite entender por qué la mayoría de las veces el diagnóstico se realiza postmortem o en el intraoperatorio de pacientes enviados para endarterectomía en contexto de TEP crónico ${ }^{1}$. El diagnóstico imagenológico con Angio-CT es sumamente difícil, por su similitud a la imagen de un trombo ${ }^{14}$, siendo de gran utilidad el PET-CT, el cual puede revelar un incremento en la captación de FDG en la arteria afectada ${ }^{15}$. Existen al menos 3 casos descritos en el cual el FAP se presenta simulando un aneurisma de la arteria ${ }^{16-18}$, situación que puede dificultar aun más la sospecha diagnóstica, como ocurrió en el caso descrito. El tratamiento se basa en la cirugía, ya sea endarterectomía, resección parcial o total de la arteria pulmonar, asociado generalmente a quimioterapia, considerando la importante frecuencia de embolización a distal ${ }^{1,10,11}$. El pronóstico en general es malo, con una sobrevida al año de $20 \%$ $y$ de $6 \%$ a los 5 años $^{19}$. Sin cirugía, se ha reportado una sobrevida media de 1,5 meses, la cual aumenta a 10 meses con resección quirúrgica ${ }^{20}$.

La paciente del caso clínico comenzó con clínica de TEP 3 años previo al diagnóstico de FAP y presentó posteriormente episodios que se interpretaron como recurrencia del mismo, a pesar de tener una adecuada anticoagulación. La aparición de hemoptisis y en relación a ello, la pesquisa de un aneurisma de la arteria interlobar izquierda hizo pensar en un síndrome de Hughes Stovin. Considerando la hemoptisis como un signo de alarma debido a la alta mortalidad de dicha complicación, se decidió la pronta realización de 
cirugía, llevándose a cabo el estudio preoperatorio sin incluir un PET-CT, examen que probablemente hubiese ayudado al diagnóstico, aunque el tratamiento hubiese sido igualmente quirúrgico. Resulta interesante la presentación de este caso simulando TEP a repetición, principal confusión diagnóstica con el FAP, a lo cual se sumó la aparición de un aneurisma de la arteria pulmonar, situación sumamente infrecuente en esta patología, pero que debe tenerse en consideración como parte del diagnóstico diferencia de un aneurisma de arteria pulmonar.

\section{Referencias}

1. Anderson MB, Kriett JM, Kapelanski DP, Tarazi R, Jamieson SW. Primary pulmonary artery sarcoma: a report of six cases, Ann Thorac Surg 1995; 59: 1487-90.

2. Tao Jin, Chong Zhang, Zhiying Feng, Yiming Ni. Case report-Pulmonary. Primary pulmonary artery sarcoma. Interactive Cardio Vascular and Thoracic Surgery 2008; 7: 722-4.

3. Khalid U, Saleem T. Hughes-Stovin Syndrome. Orphanet Journal of Rare Diseases 2011; 6: 15.

4. Bartter T, Irwin RS, Nash G. Aneurysms of the pulmonary arteries. Chest 1988; 94: 1065-75.

5. Khalil A, Parrot A, Fartoukh M, Marsault C, Carette MF. Images in cardiovascular medicine. Large pulmonary artery aneurysm rupture in Hughes-Stovin syndrome: multidetector computed tomography pattern and endovascular treatment. Circulation 2006; 114: 380-1.

6. Denecke T, Staeck O, Amthauer H, López E. PET/CT visualises inflammatory activity of pulmonary artery aneurysms in Behçet disease. Eur J Nucl Med Mol Imaging 2007; 34: 970.

7. Lee J, Noh JW, Hwang JW, Kim H, Ahn JK, Koh EM, et al. Successful cyclophosphamide therapy with complete resolution of pulmonary artery aneurysm in HughesStovin syndrome patient. Clin Rheumatol 2008; 27: 1455-8.

8. Bradbury AW, Milne AA, Murie JA. Surgical aspects of Behçet's disease. Br J Surg 1994; 81: 1712-21.

9. Ozbek C, Emrecan B, Orgen Calli A, Gurbuz A. Intimal sarcoma of the pulmonary artery with retrograde extension into the pulmonic valve and right ventricle. Tex Heart Inst J 2007; 34 (1): 119-21.

10. Jin T, Zhang C, Feng Z, Ni Y. Primary pulmonary artery sarcoma. Interact Cardiovasc Thorac Surg 2008; 7 (4): 722-4.

11. Blackmon SH, Rice DC, Correa AM, Mehran R, Putnam JB, Smythe WR, et al. Management of primary pulmonary artery sarcomas. Ann Thorac Surg 2009; 87 (3): 977-84.

12. Mattoo A, Fedullo PF, Kapelanski D, Ilowite JS. Pulmonary artery sarcoma, a case report of surgical cure and 5-year followup. Chest 2002; 122: 745-7.

13. Cox JE, Chiles C, Aquino SL, Savage P, Oaks T. Pulmonary artery sarcomas: a review of clinical and radiographic features. J Comput Assist Tomogr 1997; 21: 750-5.

14. Long SS, Johnson PT, Hruban RH, Fishman EK. CT features of pulmonary artery sarcoma: critical aid to a challenging diagnosis. Emerg Radiol 2010; 17: 153-5.

15. Chong S, Kim TS, Kim BT, Cho EY, Kim J. Pulmonary artery sarcoma mimicking pulmonary thromboembolism: integrated FDG PET/CT. AJR Am J Roentgenol 2007; 188: 1691-3.

16. Terra RM, Fernández A, Bammann RH, Junqueira JJ, Capelozzi VL. Pulmonary artery sarcoma mimicking a pulmonary artery aneurysm. Ann Thorac Surg 2008; 86: 1354-5.

17. Koch A, Mechtersheimer G, Tochtermann U, Karck M. Ruptured pseudoaneurysm of the pulmonary artery: Rare manifestation of a primary pulmonary artery sarcoma. Interact Cardiovasc Thorac Surg 2010; 10: 120-1.

18. Henzler T, Gill I, Krissak R, Schoenberg S, Fink C. Unexpected Diagnosis of Pseudoaneurysmal Pulmonary Artery Sarcoma in a Patient with Acute Chest Pain. Journal of Thoracic Oncology 2009; 4 (11): 1438-9.

19. Kondo Y, Muto A, Nishibe T, Sato M, Ando M. Primary pulmonary artery sarcoma: difficult differential diagnosis from chronic pulmonary thromboembolism. Ann Vasc Surg 2007; 21 (4): 505-7.

20. Kruger A, Borowski M, Horst ER, de Vivie P, Theissen P, Gross-Fengels W. Symptoms, diagnosis therapy of primary sarcomas of the pulmonary artery. Thoracic Cardiovasc Surg 1990; 38: 91-5. 